\title{
RETRACTED ARTICLE: Development and In Vitro-In Vivo Characterization of Chronomodulated Pulsatile Delivery Formulation of Terbutaline Sulphate by Box-Behnken Statistical Design
}

\author{
Prabhjot Singh Bajwa, ${ }^{1}$ Anurag Bhargava, ${ }^{1}$ Jaya Sharma, ${ }^{2}$ Shailesh Sharma, ${ }^{3,4}$ \\ Abhimanyu Rai Sharma, ${ }^{1}$ and Binu Sharma ${ }^{1}$
}

\begin{abstract}
This article has been retracted by the journal because the editors have clear evidence that the scientific findings in this article are unreliable. The article describes the formulation and in vitro/in vivo testing of an Optimized Pulsatile Delivery Tablet (OP1) as follows: $10 \mathrm{~mm}$ diameter press-coated Pulsatile Delivery Tablet (PDT) that contains a $6 \mathrm{~mm}$ diameter Fast-Release Core Tablet (FRCT), prepared, characterized and tested in the rabbit pharmacokinetic study. The authors have confirmed to the editors that they actually prepared and tested a "special batch" of FRCT core tablets having a diameter of $2.5 \mathrm{~mm}$ contained in $5 \mathrm{~mm}$ press-coated PDT tablets having a diameter of $5 \mathrm{~mm}$, but the use of this smaller FRCT/PDT is not described anywhere in the manuscript. Therefore, the editors believe that since the article does not describe the dosage form that was actually studied, the findings in the article are unreliable.
\end{abstract}

All authors agreed to this retraction.

The online version of this article contains the full text of the retracted article as electronic supplementary material.

Electronic supplementary material The online version of this article (https://doi.org/10.1208/s12249-017-0838-6) contains supplementary material, which is available to authorized users.

${ }^{1}$ Ch. Devilal College of Pharmacy, Jagadhri, Yamunanagar, Haryana, India.

${ }^{2}$ School of Pharmaceutical Sciences, Jaipur National University, Jaipur, Rajasthan, India.

${ }^{3}$ Department of Pharmaceutics, Amar Shaheed Baba Ajit Singh Jujhar Singh Memorial College of Pharmacy, Bela, Ropar, Punjab, India.

${ }^{4}$ To whom correspondence should be addressed. (e-mail: drshailesh.bela@gmail.com) 